\title{
Efeito do Treinamento Físico e da Desnutrição Durante a Gestação Sobre os Eixos Cranianos de Ratos Neonatos
}

\author{
Effects of Physical Training and Malnutrition During \\ Pregnancy on the Skull Axis of Newborn Rats
}

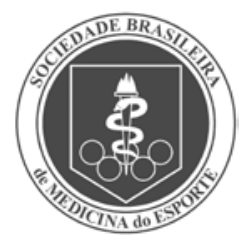

Artigo Original

\author{
Marco Fidalgo \\ Érika Michelle Correia de Macêdo² \\ Renata Dantas ${ }^{2}$ \\ Patrícia Foerster ${ }^{2}$ \\ Caroline $\mathrm{Neves}^{2}$ \\ Raul Manhães-de-Castro ${ }^{2}$ \\ Carol Góis Leandro'
}

1. Núcleo de Educação Física e Ciências do Esporte, Centro Acadêmico de Vitória, Universidade Federal de Pernambuco, Brasil.

2. Departamento de Nutrição, Universidade Federal de Pernambuco, Brasil.

Endereço para correspondência: Rua Alto do Reservatório, s/n Bela Vista 55608-680 - Vitória de Santo Antão, PE - Brasil

E-mail: marcofidalgo1@hotmail.com

\begin{abstract}
RESUMO
Nos períodos críticos de desenvolvimento do organismo, estímulos ambientais como o exercício físico e a dieta podem influenciar o fluxo placentário e o crescimento somático fetal. O objetivo do presente estudo foi avaliar as repercussões do treinamento físico e da desnutrição durante a gestação sobre os eixos cranianos de ratos neonatos. Ratos machos Wistar foram divididos de acordo com a manipulação de suas mães: não treinados controle $\left(C_{f}, n=25\right)$, treinados $\left(T_{f}, n=25\right)$, não treinados e desnutridos $\left(D_{f}, n=25\right)$, treinados e desnutridos ( $T D_{f}, n=25$ ). Mães treinadas ( $T$ e TD) foram submetidas a oito semanas de treinamento físico moderado antes e durante a gestação ( $60 \mathrm{~min} / \mathrm{dia}$, cinco dias $/ \mathrm{sem}$ a $65 \%$ do $\mathrm{VO}_{2 \text { max }}$ ). Mães desnutridas ( $\mathrm{D}$ e TD) receberam dieta hipoproteica durante a gestação (8\% caseína) enquanto as nutridas (C e T) receberam dieta normoproteica (17\% caseína). No primeiro dia pós-natal foi verificado número de filhotes nascidos por ninhada, peso da ninhada e peso ao nascer, eixo látero-lateral do crânio (ELLC) e anteroposterior do crânio (EAPC), eixo longitudinal do corpo (EL) e comprimento da cauda (CC) de cada neonato. No terceito dia pósparto, os encéfalos foram extraídos e pesados. Durante a gestação, as fêmeas dos grupos T e D apresentaram menor ganho de peso em comparação ao grupo $C$ na terceira semana $(C=34,4 \pm 1,3 ; T=30,7 \pm 0,60 ; D$ $=25,8 \pm 0,78 ; \mathrm{TD}=29,9 \pm 0,83)$. Os grupos desnutridos apresentaram menor peso ao nascer em relação aos seus respectivos controles $\left(C_{f}=6,3 \pm 0,1 ; T_{f}=6,3 \pm 0,1 ; D_{f}=4,7 \pm 0,07 ; D_{f}=5,0 \pm 0,06\right)$. O grupo $D_{f}$ apresentou as medidas do ELLC $\left(C_{f}=9,8 \pm 0,06 ; T_{f}=9,8 \pm 0,05 ; D_{f}=9,2 \pm 0,04 ; D_{f}=9,6 \pm 0,13\right)$ e EAPC $\left(C_{f}\right.$ $\left.=18,1 \pm 0,1 ; T_{f}=18,2 \pm 0,1 ; D_{f}=17,5 \pm 0,1 ; T_{f}=18,0 \pm 0,2\right)$ menores comparadas aos filhotes controles. Com base nos resultados, pode-se concluir que a desnutrição no período fetal alterou o desenvolvimento somático enquanto o treinamento físico influenciou positivamente os eixos do crânio dos conceptos.
\end{abstract}

Palavras-chave: craniometria, esforço físico, desnutrição proteica, desenvolvimento fetal.

\begin{abstract}
In critical periods of body development, environmental stimuli such as physical exercise and diet may influence on placental flow and fetal somatic growth. The aim of this study was to evaluate the effects of physical training and malnutrition during pregnancy on the skull axis of newborn rats. Male Wistar rats were divided according to manipulation of their mothers: untrained control $\left(C_{f}, n=25\right)$, trained $\left(T_{f}, n=\right.$ 25), untrained and malnourished ( $\left.M_{f}, n=25\right)$, trained and malnourished (TMf, $\left.n=25\right)$. Trained mothers ( $T$ and TM) were submitted to 8 weeks of moderate physical training before and during pregnancy (60min/ day, 5 days/wk to $65 \%$ of $\mathrm{VO}_{\text {max }}$ ). Malnourished mothers ( $\mathrm{M}$ and TM) received a low protein diet during pregnancy ( $8 \%$ casein) while the nourished ( $C$ and $T$ ) were fed with normal diet (17\% casein). On the 1 st postnatal day, the number of pups born per litter, litter weight and birth weight, latero-lateral axis of skull (LLAS) and antero-posterior axis of skull (APAS), longitudinal axis of the body (LA) and length of tail (LT) of each neonate were verified. On the 3rd day after delivery, the brains were extracted and weighed. During pregnancy, the females of the $T$ and $M$ groups showed lower weight gain compared with group $C$ at $3 r d$ week $(C=34.4 \pm 1.3, T=30.7 \pm 0.60, M=25.8 \pm 0.78, T M=29.9 \pm 0.83)$. The malnourished group had lower birth weight in relation to their respective controls $\left(C_{f}=6.3 \pm 0.1, T_{f}=6.3 \pm 0.1, M_{f}=4.7 \pm 0.07 ; \mathrm{TM}_{f}\right.$ $=5.0 \pm 0.06)$. Group $M_{f}$ presented measures of $\operatorname{LAS}\left(C_{f}=9.8 \pm 0.06, T_{f}=9.8 \pm 0.05, M_{f}=9.2 \pm 0.04 ; M_{f}=\right.$ $9.6 \pm 0.13)$ and EAPC $\left(C_{f}=18.1 \pm 0.1, T_{f}=18.2 \pm 0.1, M_{f}=17.5 \pm 0.1, T M_{f}=18.0 \pm 0.2\right)$ lower compared to controls. Based on the results, it can be concluded that malnutrition during fetal period changed somatic development, while physical training positively influenced on the skull axis of the concepts.
\end{abstract}

Keywords: craniometry, physical exertion, protein malnutrition, fetal development. 


\section{INTRODUÇÃO}

Durante a vida fetal, neonatal e infância, tecidos e órgãos apresentam períodos críticos para sua maturação e desenvolvimento(1). Nestes períodos, estímulos ambientais, como estresse, álcool, fumo, fármacos, hipóxia, nutrição e atividade física influenciam na estruturação dos sistemas fisiológicos ${ }^{(2-5)}$. Destes estímulos, a nutrição e o estilo de vida da mãe são determinantes para o desenvolvimento de padrões metabólicos e crescimento somático do feto(5).

Vários estudos têm verificado uma relação entre a nutrição no período fetal e neonatal e o crescimento e desenvolvimento orgânico ${ }^{(6-8)}$. Em ratos, uma dieta deficiente (8\% de proteína) resultou em alteração no desenvolvimento e tamanho do cérebro, no número de neurônios, aprendizagem e memória, na ontogênese de reflexos e no padrão de atividade locomotora ${ }^{(6,9,10)}$. $O$ aporte inadequado de nutrientes e oxigênio pode ser o principal mecanismo que acarreta prejuízos ao crescimento feto-placentário ${ }^{(5)}$.

Por outro lado, o exercício físico durante a gestação pode ter repercussões positivas no desenvolvimento fetal(11). Contudo, o benefício do exercício físico vai depender do momento da gestação e da magnitude do esforço (intensidade, duração e frequência das sessões de exercício) ${ }^{(12,13)}$. Em humanos, estudos apontam que exercícios físicos de intensidade moderada iniciados nos primeiros estágios da gestação podem aumentar o fluxo sanguíneo placentário com repercussões positivas sobre o crescimento intrauterino ${ }^{(5,14)}$. Por outro lado, os exercícios físicos intensos e altas rotinas diárias de atividade física durante a gestação estão associados com baixo peso ao nascer ${ }^{(12,15,16)}$. Em animais, ratas exercitadas em esteira ergométrica $\left(20 \mathrm{~m} / \mathrm{min}\right.$, duração de 20 a 70 minutos/dia, cinco dias/semana, a $10^{\circ}$ de inclinação) apresentaram menores níveis de insulina plasmática e o peso fetal e da ninhada não foram afetados ${ }^{(17)}$. No sistema nervoso, Parnpiansil et al. (2003) $)^{(18)}$ verificaram que em filhotes de ratas submetidas a um protocolo de treinamento em esteira $(20 \mathrm{~m} / \mathrm{min}$, $30 \mathrm{~min} / \mathrm{dia}$, cinco dias/semana, com aproximadamente $70 \%$ do $\mathrm{VO}_{2 \max }$ ) durante a gestação, houve aumento na expressão de mRNA de fatores neurotróficos derivados do cérebro (BDNF) no hipocampo cerebral e maior habilidade na aprendizagem motora espacial. Ainda, Amorim et al. (2009) ${ }^{(19)}$ constataram que o treinamento físico moderado (1h/ dia, cinco dias/semana, durante sete semanas, com aproximadamente $\left.65 \% \mathrm{VO}_{2 \max }\right)$ não alterou o consumo de oxigênio de repouso de ratas treinadas e desnutridas em relação aos animais controles e repercutiu positivamente na taxa de crescimento de sua prole até os 90 dias.

Uma vez que a desnutrição fetal está associada a um déficit no desenvolvimento fetal e que o treinamento físico de intensidade moderada pode atenuar estas alterações, o presente estudo tem como objetivo investigar a influência do treinamento físico e da desnutrição gestacional sobre os eixos corporais, comprimento da cauda e o peso do encéfalo de ratos neonatos.

\section{MATERIAL E MÉTODOS}

Foram utilizadas 20 ratas albinas Wistar primíparas (peso corporal = 200 - 15g) provenientes da colônia do Departamento de Nutrição da Universidade Federal de Pernambuco. Os animais foram mantidos em biotério com temperatura de $23-2^{\circ} \mathrm{C}$, e ciclo claro/escuro (claro das 21:00 às 09:00h, escuro das 9:00 às 21:00h). Até o período do acasalamento, as ratas receberam dieta padrão de biotério (52\% carboidrato, $21 \%$ proteína e $4 \%$ lipídios - Ração Labina ${ }^{\circledR}$, Agribrands-Purina Ltda, Paulínia, SP, Brasil) e água ad libitum. Este estudo foi aprovado pelo Comitê de Ética em Experimentação Animal do Centro de Ciências Biológicas da Universidade Federal de Pernambuco e seguiu as normas sugeridas pelo Comitê Brasileiro de Experimentação Animal (COBEA) - nº 23076.000312/2006-39.
As ratas foram inicialmente divididas em dois grupos: não treinado controle $(C, n=10)$ e treinado $(T, n=10)$. O grupo $T$ foi submetido a um protocolo de treinamento físico moderado (60 minutos/dia, cinco dias/semana, durante oito semanas, com intensidade de aproximadamente $65 \%$ do $\mathrm{VO}_{2 \text { max }}$ em esteira ergométrica adaptada para ratos (Insight ${ }^{\circledR}-$ SP, Brasil).

Após cinco semanas de treino, as ratas foram acasaladas com machos da mesma linhagem (duas ratas para cada um rato). Foi considerado como provável dia da concepção a visualização de espermatozoides em lâmina, em microscópio de luz, após coleta do esfregaço vaginal(20). Assim, metade dos animais de cada grupo recebeu dieta normoproteica (caseína a 17\%) e a outra metade recebeu dieta hipoproteica (caseína a 8\%), sendo formados mais dois grupos experimentais: não treinado controle $(C, n=5)$, treinado $(T, n=5)$ e não treinado desnutrido $(D, n=5)$, treinado desnutrido ( $T-D, n=5)$. Os grupos $T$ mantiveram o programa de treinamento, com duração e intensidade progressivamente reduzida (primeira semana $=50 \mathrm{~min} / \mathrm{dia}$, segunda semana $=$ $30 \mathrm{~min} / \mathrm{dia}$, terceira semana $=20 \mathrm{~min} / \mathrm{dia} ;$ cinco dias $/$ semana, durante oito semanas, com intensidade de aproximadamente $40 \%$ do $\left.\mathrm{VO}_{2 \max }\right)$ até $019^{\circ}$ dia de gestação. Após o nascimento, ninhadas com seis fiIhotes machos foram divididos em quatro grupos de acordo com a manipulação de suas mães (CCCCCCCT $T_{f}, \mathrm{n}=25$, provenientes de mães $T_{;} D_{f}, n=25$, provenientes de mães $D_{;}$e $T D_{f}, n=25$, provenientes de mães TD). Na lactação, todas as ratas receberam dieta à base de caseína a $17 \%$ até o terceiro dia pós-parto.

\section{Avaliação do peso corporal}

As ratas foram pesadas semanalmente em balança eletrônica (Marte $^{\circledast}$, modelo S-4000, com sensibilidade de 0,1g-MG, Brasil), no período pré-gestacional, e diariamente, na gestação. O percentual de ganho de peso corporal das mães foi calculado tendo como base o peso do primeiro dia de avaliação, segundo a fórmula:

$\%$ ganho de peso $=[$ peso do dia $(\mathrm{g}) \times 100 /$ peso do 1으 dia (g) $]-100$

No nascimento, foi verificado o número de filhotes nascidos por ninhada, peso da ninhada (g) e peso corporal (g) de cada neonato.

\section{Avaliação murinométrica e extração dos encéfalos}

No primeiro dia pós-natal, foram determinadas as seguintes medidas (em milímetros - mm) dos filhotes de acordo com as técnicas murinométricas sugeridas por Barros et al.(6) e Silva et al.(8):a) eixo látero-lateral do crânio (ELLC), linha perpendicular ao eixo longitudinal do crânio a qual divide ao meio os pavilhões auriculares; b) eixo anteroposterior do crânio (EAPC), linha média da extremidade do focinho à região externa da crista occipital; c) eixo longitudinal do corpo (EL), distância entre a extremidade do focinho à extremidade posterior do corpo; d) comprimento da cauda (CC), distância entre a extremidade posterior do corpo à extremidade da cauda. As medidas foram realizadas com um paquímetro com acurácia de $0,01 \mathrm{~mm}$ (Starret ${ }^{\circledR}$-SP, Brasil).

No terceiro dia após o nascimento, os neonatos foram sacrificados por decaptação e os encéfalos foram extraídos, lavados em solução de $\mathrm{NaCl}$ a 0,9\%e pesados em balança eletrônica (Marte ${ }^{\circledR}$, modelo AL 500, com sensibilidade de 0,001g - MG, Brasil).

\section{Análise estatística}

Para comparação de parâmetros das ratas durante o treinamento foi utilizado o t-test Student. Coeficiente de correlação de Pearson foi utilizado para correlacionar ganho de peso na gestação com número de filhotes nascidos por mãe. Para comparação entre os grupos experimentais formados foi utilizada ANOVA two-way, de medidas repetidas, seguida do teste post-hoc de Bonferroni para 
comparação múltipla. Os valores estão expressos em média e erro padrão da média. O nível de significância foi mantido em $5 \%(p<0,05)$ em todos os casos. As análises dos dados foram realizadas através do programa estatístico Graphpad Prisma ${ }^{\circledR}$ (versão 4.0).

\section{RESULTADOS}

Após as cinco semanas que precederam a concepção, o ganho de peso corporal de ambos os grupos foi semelhante $(C=10,2 \%$ eT $=8,6 \%$, $p>0,05)$. Durante a gestação, as fêmeas dos grupos T e D apresentaram menor ganho de peso em comparação ao grupo C na terceira semana (figura 1). Dados foram ajustados para o número de filhotes nascidos de cada mãe [C, 12 (9-13);T, 10 (8-14); D, 11 (7-12); eTD, 12 (7-12); os valores estão expressos como mediana (mínimo e máximo)], e o coeficiente de correlação entre o número de filhotes nascidos e o ganho de peso corporal das mães não foi significante $\left(r^{2}=0,16 ; p=0,485\right)$.

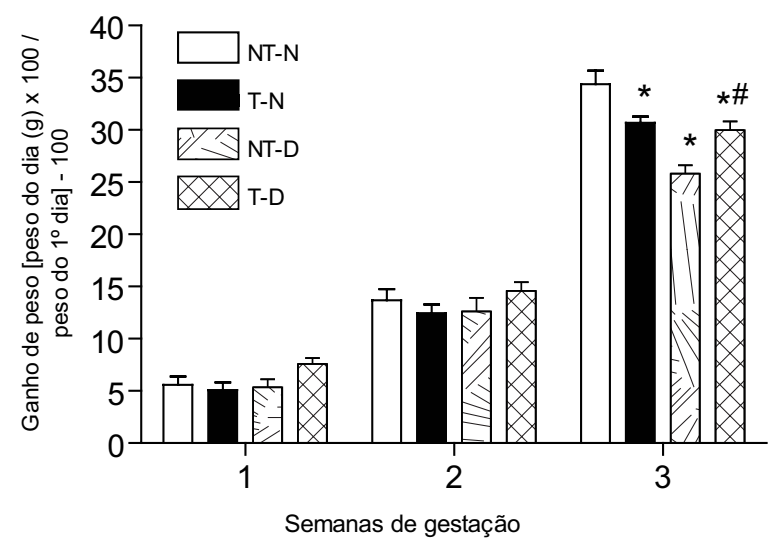

Figura 1. Ganho de peso corporal das mães durante as três semanas de gestação. Grupos: NT-N ( $n=5)$; T-N ( $n=5) ;$ NT-D ( $n=5)$ e T-D ( $n=5)$. Os dados estão representados em médiatEPM. * $\mathrm{p}<0,05$ vs NT-N, \#p<0,05 vs NT-D. ANOVA two-way, de medidas repetidas, seguida do Teste de Bonferroni.

Os filhotes provindos de mães submetidas à desnutrição na gestação apresentaram um menor peso corporal ao nascer em relação aos seus respectivos controles (figura 2).

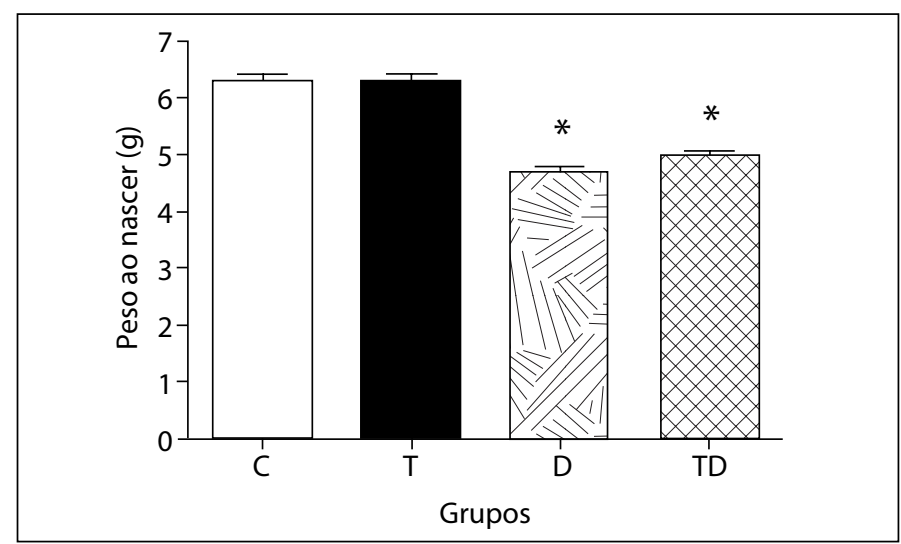

Figura 2. Peso ao nascer dos filhotes. Grupos: $C_{f}(n=25) ; T_{f}(n=25) ; D_{f}(n=25)$ e $\mathrm{TD}_{\mathrm{f}}(\mathrm{n}=25)$. Os dados estão representados em média \pm EPM. ${ }^{*} p<0,05$ vs. C. ANOVA two-way, de medidas repetidas, seguida do Teste de Bonferroni.

Quanto ao tamanho dos eixos corporais dos filhotes, os animais do grupo $D_{f}$ apresentaram as medidas do ELLC e EAPC menores comparadas aos filhotes controles (figura 3). Não houve diferença para o EL (NT$\left.N_{f}=47,1 \pm 0,18 ; T-N_{f}=47,2 \pm 0,60 ; N T-D_{f}=46,2 \pm 0,28 ; T-D_{f}=46,7 \pm 0,58\right), C C$ $\left(\mathrm{NT}-\mathrm{N}_{f}=15,5 \pm 0,12 ; \mathrm{T}-\mathrm{N}_{f}=15,8 \pm 0,26 ; \mathrm{NT}-\mathrm{D}_{f}=14,9 \pm 0,15 ; \mathrm{T}-\mathrm{D}_{f}=15,3 \pm 0,18\right)$

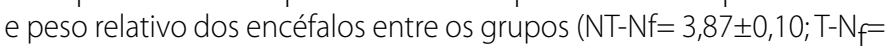
$4,31 \pm 0,31 ; N T-D_{f}=4,42 \pm 0,17 ; T-D_{f}=3,81 \pm 0,08$ ).
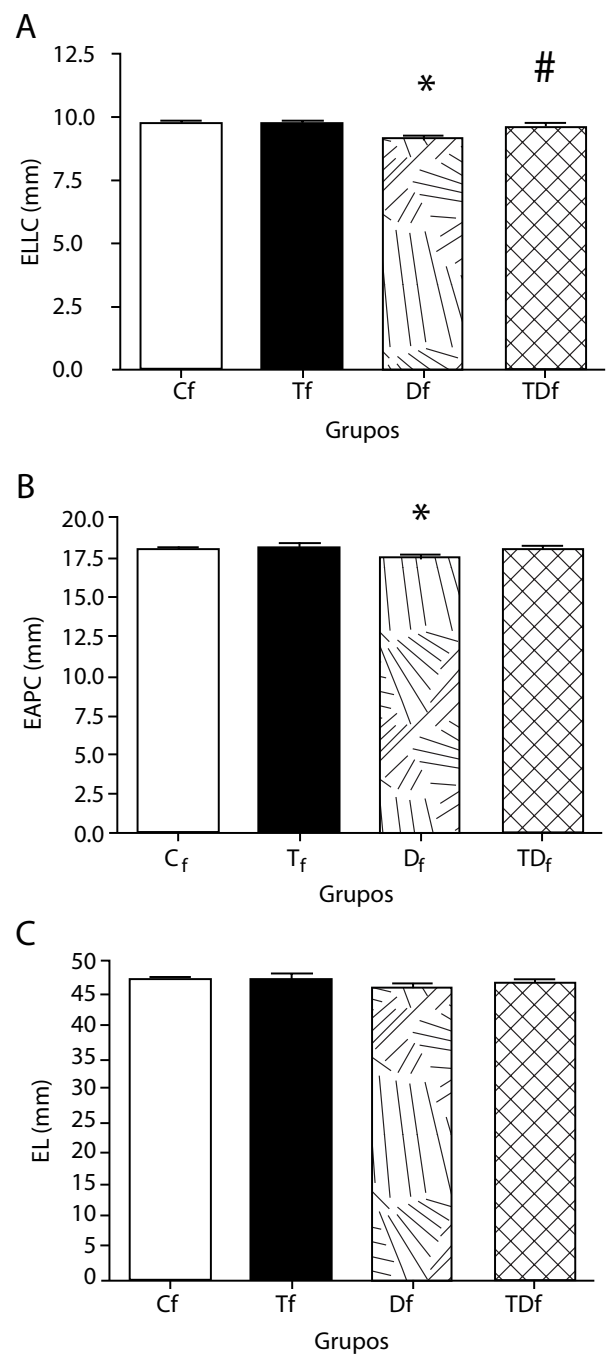

Figura 3. Tamanho do ELLC, EAPC e EL dos filhotes. Grupos: $C_{f}(n=25) ; T_{f}(n=25) ; D_{f}(n$ $=25)$ e $\mathrm{TD}_{\mathrm{f}}(\mathrm{n}=25)$. Os dados estão representados em média $\pm \mathrm{EPM}$. ${ }^{*} \mathrm{p}<0,05 \mathrm{vs}$. $\mathrm{C}$, \#p $<0,05$ vs. D. ANOVA two-way, de medidas repetidas, seguida do Teste de Bonferroni.

\section{DISCUSSÃO}

O presente estudo teve como objetivo avaliar a influência de dois estímulos ambientais, treinamento físico e desnutrição, sobre os eixos cranianos de filhotes ao nascer. A desnutrição, quando aplicada no início da vida, pode estar associada à déficits no crescimento e desenvolvimento ${ }^{(6-7)}$. Por outro lado, o treinamento físico leve e moderado durante a gestação tem sido considerado um estímulo positivo para o crescimento feto-placental(5,12).

O ganho de peso materno durante a gestação tem por finalidade acumular reservas energéticas para o desenvolvimento embrionário(5). Em relação à desnutrição durante a gestação, nossos resultados corroboram dados encontrados na literatura, os quais verificaram que o uso de dietas hipoproteicas (8\% de caseína) e restrição de dieta controle (50\% da quantidade) induziram déficits no peso corporal das mães ${ }^{(6-7)}$.

Exercícios físicos regulares durante a gestação podem ser estratégias efetivas para o controle no ganho de peso ${ }^{(13,21)}$. No presente estudo, o treinamento físico durante a gestação induziu redução no ganho de peso corporal materno. Em contraste, Treadway et al. (1986) (23) observaram que ratas submetidas somente ao treino $(31 \mathrm{~m} / \mathrm{min}$, 2h/dia, cinco dias/semana, oito semanas; durante a gestação: $27 \mathrm{~m} /$ min, 1h/dia) apresentaram maior ganho de peso durante a gestação. Nossos achados fornecem indícios de que o treinamento no período gestacional possa ter induzido ganho de massa muscular e redução de tecido adiposo ${ }^{(11,13)}$. 
A disponibilidade de nutrientes é essencial para manutenção da função placentária e formação fetal. Ozanne e Hales(24) associam a desnutrição precoce com uma redução dos depósitos maternos, comprometimento no fluxo feto-placentário e na taxa de divisão celular nos tecidos e órgãos dos fetos. Estudos adotando modelo de restrição na quantidade da dieta verificaram redução no peso corporal ao nascer, no tamanho do neonato e no peso de órgãos ${ }^{(1,7,22)}$. Por sua vez, o exercício físico induz flutuações intermitentes na oferta de substratos. Desta forma, é produzido um estímulo recorrente que, mesmo diante da privação, ocorre difusão de nutrientes e oxigênio para o feto(5). Amorim et al. ${ }^{(19)}$ verificaram que o treinamento físico moderado atenuou os impactos de uma dieta hipoproteica sobre o consumo de oxigênio de repouso de ratas durante a gestação e aumentou a taxa de crescimento de sua prole até os 90 dias. Exercícios físicos moderados realizados durante a gestação podem estimular mecanismos que incluem aumento do aporte de nutrientes e oxigênio para o feto, através do incremento no fluxo placentário, repercutindo no peso ao nascer ${ }^{(5)}$.

Estudos prévios demonstram que a desnutrição fetal e neonatal pode alterar o tamanho dos eixos cranianos e crescimento cerebral|(6,8). Avaliação das medidas cranianas é muito utilizada em estudos com humanos e experimentais e apresenta relação direta com insultos nutricionais precoces e o desenvolvimento do sistema nervoso ${ }^{(25,26)}$. Além do peso como indicador de complicações intrauterinas, a verificação de medidas craniofaciais é fundamental para identificação da existência de anormalidades, como redução da massa cerebral, do comprometimento no desenvolvimento neuromotor e dos processos que influenciam a gênese de patologias na idade adulta ${ }^{(25,26)}$. Nossos resultados apontam que a desnutrição fetal foi determinante para diminuição no tamanho dos eixos cranianos. Silva et al. ${ }^{(8)}$, utilizando modelo de desnutrição através de dieta com 8\% de proteína (DBR - dieta básica regional) observaram também redução nas medidas dos animais desnutridos. Sobre o desenvolvimento do sistema nervoso, Bennis-Taleb e Remacle ${ }^{(27)}$, submetendo ratas a dieta hipoproteica (8\% de proteína) durante a gestação, observaram, na prole, alteração na densidade de

\section{REFERÊNCIAS}

1. Desai M, Gayle D, Babu J, Ross,MG. Permanent reduction in heart and kidney organ growth in offspring of undernourished rat dams. Am J Obstet Gynecol. 2005;193:1224-32.

2. Kumsta R, Entringer S, Hellhammer DH, Wüst S. Cortisol and ACTH responses to psychosocial stress are modulated by corticosteroid binding globulin levels. Psychoneuroendocrinology. 2007;32:1153-7.

3. Lopes de Souza S, Nogueira MI, Deiro TCBJ, Manhães-de-Castro FM, Mendes-da-Silva C, Silva MC, et al. Differential effects on somatic and reflex development by chronic clomipramine treatment. Physiol Behav. 2004;82:375-9.

4. Strauss RS. Effects of the intrauterine environment on childhood growth. Br Med Bull. 1997;33:81-95.

5. Clapp JF. Influence of endurance exercise and diet on human placental development and fetal growth. Placenta. 2006;27:527-34

6. Barros KMFT, Manhães-de-Castro R, Lopes-de-Souza S, Matos RJB, Deiró TCBJ, Cabral-Filho JE, et al. A regional model (Northeastern Brazil) of induced malnutrition delays ontogeny of reflexes and locomotor activity in rats. Nutr Neurosci. 2006;9:99-104.

7. Desai M, Crowtherl NJ, Lucas A, Hales N. Organ-selective growth in the offspring of protein-restricted mothers. Br J Nutr. 1996;76:591-603.

8. Silva HJ, Marinho SMOC, Toscano AE, Albuquerque CG, Moraes SRA, Manhães-de-Castro R. Protocol of mensuration to avaliation of indicators of somatic development of Wistar rats. Int J Morphol. 2005;23:227-30.

9. Gressens P, Muaku SM, Besse L, Nsegbe E, Gallego J, Delpech B, et al. Maternal protein restriction early in rat pregnancy alters brain development in the progeny. Brain Res Dev Brain Res. 1997;103:21-35.

10. Do Monte-Silva KK, Assis FL, Leal GM, Guedes RC. Nutrition-dependent influence of peripheral electrical stimulation during brain development on cortical spreading depression in weaned rats. Nutr Neurosci. 2007;10:187-94.

11. Clapp III JF. The effects of maternal exercise on fetal oxygenation and feto-placental growth. Eur J Obstet Gynecol Reprod Biol. 2003;110:S80-5.

12. Clapp III JF, Kim H, Burciu B, Schmidt S, Petry K, Lopez B. Continuing regular exercise during pregnancy: Effect of exercise volume on fetoplacental growth. Am J Obstet Gynecol. 2002;186:142-7.

13. Leitão MB, Lazzoli JK, Oliveira MAB, Nóbrega ACL, Silveira GG, Carvalho T, et al. Posicionamento Oficial da Sociedade Brasileira de Medicina do Esporte: Atividade Física e Saúde na Mulher. Rev Bras Med Esporte. 2000;6:215-20.

14. Clapp III JF, Kim H, Burciu B, Lopez B. Beginning regular exercise in early pregnancy:Effect on fetopla- vasos sanguíneos do córtex cerebral no terceiro dia pós-parto e redução na vascularização na idade adulta.

Escassos são os estudos que verificam a influência do exercício sobre o desenvolvimento do sistema nervoso e medidas cranianas. Entretanto, parece que o exercício físico é efetivo para proteger o cérebro. Parnpiansil et al. ${ }^{(18)}$, verificando a influência do exercício físico sobre o desenvolvimento do encéfalo, observaram em filhotes de ratas exercitadas em esteira aumento na expressão de mRNA de BDNF. No presente estudo, o protocolo de treino utilizado parece ter sido eficiente, pois os filhotes do grupo treinado e treinado desnutrido não apresentaram diferenças no tamanho dos eixos cranianos em relação aos animais controle. Se o treinamento físico durante a gestação aumenta o fluxo placentário e a oferta de nutrientes e oxigênio para o feto ${ }^{(5)}$, a hipótese que tenha ocorrido preservação das estruturas craniofaciais por um mecanismo de neuroproteção parece plausível(28).

Conforme nossos achados, é possível que o treinamento de intensidade leve a moderada possa atenuar as consequências da desnutrição no período crítico do desenvolvimento. Pelo que já foi aventado, parece que a maior irrigação feto-placentária e disponibilidade de nutrientes e oxigênio neste período, proporcionada pelo aumento da demanda em consequência do esforço, é favorável para programar o crescimento intrauterino. A relevância do tema aponta a necessidade de continuidade nas investigações, verificando a influência do treinamento materno associado à dieta sobre a neuroplasticidade e o desenvolvimento neuromotor destes animais em diferentes idades.

\section{AGRADECIMENTOS}

Ao Conselho Nacional de Desenvolvimento Científico e Tecnológico (CNPq) e à Coordenação de Aperfeiçoamento de Pessoal de Nível Superior (CAPES) por tornarem possível a realização deste estudo.

Todos os autores declararam não haver qualquer potencial conflito de interesses referente a este artigo. cental growth. Am J Obstet Gynecol. 2000;183:1484-8.

15. ACOG Committee Opinion. Exercise during pregnancy and the postpartum period. Obstet Gynecol. 2002;99:171-3.

16. Rao S, Kanade A, Margetts BM, Yajnik CS, Lubree H, Rege S, et al. Maternal activity in relation to birth size in rural India. The Pune Maternal Nutrition Study. Eur J Clin Nutr. 2003;57:531-42.

17. López-Luna P, Iglesias MA, Muñoz C, Herrera E. Aerobic exercise during pregnancy reverts maternal insulin resistance in rats. Med Sci Sports Exerc. 1998;30:1510-4.

18. Parnpiansil P, Jutapakdeegul N, Chentanezb T, Kotchabhakdi N. Exercise during pregnancy increases hippocampal brain-derived neurotrophic factor mRNA expression and spatial learning in neonatal rat pup. Neurosci Lett. 2003;352:45-8.

19. Amorim MF, Santos JA, Hirabara SM, Nascimento E, Lopes-de-Souza S, Manhães-de-Castro R, et al. Can physical exercise during gestation attenuate the effects of a maternal perinatal low-protein diet on oxygen consumption in rats? Exp Physiol. 2009;94:906-13.

20. Marcondes FK, Bianchi FJ, Tanno AP. Determination of the estrous cycle phases of rats: some helpful considerations. Braz J Biol, 2002;62:609-14.

21. Artal R, O'Toole M, White S. Guidelines of the American College of Obstetricians and Gynecologists for exercise during pregnancy and the postpartum period. Br J Sports Med. 2003;37;6-12.

22. Araya J, Reyes MC, Baginsky CM, Ruz M. Cellular growth of uterus, placenta and fetus during chronic maternal caloric restriction in rats. Arch Latinoam Nutr. 1983;33:814-25.

23. Treadway J, Dover EV, Morse W, Newcomer L, Craig BW. Influence of exercise training on maternal and fetal morphological characteristics in the rat. J Appl Physiol. 1986;60:1700-3.

24. Ozanne $\mathrm{SE}$, Hales $\mathrm{CN}$. The long-term consequences of intra-uterine protein malnutrition for glucose metabolism. Proc Nutr Soc. 1999;58:615-9.

25. Graves AB, Mortimer JA, Larson EB, Wenzlow A, Bowen JD, McCormick WC. Head circumference as a measure of cognitive reserve - Association with severity of impairment in Alzheimer's Disease. $\mathrm{Br} J$ Psychiatry. 1996;169:86-92.

26. Barker DJ, Osmond C, Simmonds SJ, Wield GA. The relation of small head circumference and thinness at birth to death from cardiovascular disease in adult life. BMJ. 1993;306:422-6.

27. Bennis-Taleb N, Remacle C. A low-protein isocaloric diet during gestation affects brain development and alters permanently cerebral cortex blood vessels in rat offspring. J Nutr. 1999;129:1613-9.

28. Dishman RK. The new emergence of exercise neurobiology. Scand J Med Sci Sports. 2006;16:379-80. 